\title{
Educando para el desarrollo y la ciudadanía global a través de la psicología positiva
}

\author{
Noelia Santamaría-Cárdaba. Universidad de Valladolid \\ Recepción: 28.02.2018| Aceptado: 19.03.2018 \\ Correspondencia a través de ORCID: Noelia Santamaría-Cárdaba iD 0000-0001-6864-9330 \\ Citar: Santamaría-Cárdaba, N. (2018). Educando para el desarrollo y la ciudadanía global a \\ través de la psicología positiva. ReiDoCrea, 7, 98-109.
}

\begin{abstract}
Resumen: La educación centrada en la formación en valores y en la creación de ciudadanos globales es uno de los temas a los que se debería dar una especial prioridad desde los centros educativos. Método: en este estudio se comprueba si a través del proyecto de psicología positiva Aulas Felices se potencia la formación de ciudadanos críticos. La metodología empleada fue cualitativa, pues se empleó como técnica de recolección de datos la observación participante. Resultados: Las actividades propuestas basadas en el programa Aulas Felices funcionaron adecuadamente y despertaron en el estudiantado valores como la solidaridad, el respeto y la cooperación. Conclusión: Se debe educar ciudadanos globales y libres en los centros educativos de forma transversal para que el alumnado sea capaz de entender el mundo en el que viven y cambiarlo hacia uno más justo y solidario.
\end{abstract}

Palabras clave: Educación | Psicología Positiva

Educating for Development and Global Citizenship through Positive Psychology

\begin{abstract}
Education focused on the teaching of values and the creation of global citizens is one of the issues that should be given special priority in schools. Method: This study analyzes if, through the positive psychology project Happy Classrooms, the training of critical citizens is promoted. The methodology was qualitative, since participant observation was used as a data collection technique. Results: The proposed activities based on the Happy Classrooms program worked properly and aroused in the students values such as solidarity, respect and cooperation. Conclusion: Educating global and free citizens should be a cross-curricular theme in schools so that students are able to understand the world in which they live and make it a more fair and supportive one.
\end{abstract}

Keywords: Education | Positive Psychology

\section{Introducción}

La formación en valores es una cuestión importante en la sociedad actual pues, como afirman autores como Díaz (2005) o Davies, Evans y Reid (2005), es uno de los temas en los que se está centrando en los últimos años el debate educativo. De este modo, resulta necesario que se trate de forma transversal en los centros escolares la Educación para el Desarrollo y la Ciudadanía Global (en lo sucesivo, EDCG). El presente estudio analiza una propuesta de desarrollo de la ciudadanía global a través de la psicología positiva, concretamente, mediante el programa Aulas Felices elaborado por Arguís, Bolsas, Hernández y Salvador (2012).

La noción de Educación para el Desarrollo, según las generaciones planteadas por Mesa (2000), ha ido evolucionando partiendo en los años 40 de un enfoque que abogaba por la caridad y la asistencia a los países del Sur ayudándoles mediante campañas de sensibilización y recaudación de fondos (Boni y León, 2013) hasta llegar en torno a 1990 a la quinta generación denominada EDCG o cosmopolita. Este quinto enfoque de EDCG promueve un modelo de vida social fundamentado en los principios de solidaridad, justicia y participación ciudadana que tiene como finalidad conseguir una vida feliz para las personas. Actualmente, autores como Calvo (2017) o Sainz de Murieta (2016) afirman que se encuentra en vigor una sexta generación que ampliaría 
el sistema de los cinco enfoques planteado por Mesa (2000) y fundamentado por autores como Argibay y Celorio (2005), Boni (2014), u Ortega (2007).

Esta propuesta toma como referencia las definiciones de EDCG establecidas por Celorio (2017), Celorio y Celorio (2011), Mesa (2014), Smith et al. (2016) y Starkey (2012) para establecer el significado de EDCG. De esta forma, se comprende la EDCG como un proceso educativo que quiere desarrollar en las personas su pensamiento crítico y su autonomía para que entiendan los problemas que acucian a la realidad social y, especialmente, a las relaciones entre países del Norte y del Sur. La finalidad de la EDCG es potenciar una ciudadanía crítica y global que defienda un fuerte compromiso con los Derechos Humanos para tratar de convertir el mundo en un lugar más justo y más humano.

Los ciudadanos globales deben adquirir mayor responsabilidad social y ser conscientes de los problemas que afectan al mundo, de las causas que generan la pobreza y la desigualdad para tratar de actuar mejorando el bienestar de las zonas más desfavorecidas (Monk 2014; Murray 2006). Reysen y Katzarska-Miller (2013) añaden la importancia de comprender la diversidad cultural existente en el mundo y de adquirir una capacidad de defender la justicia social. Andreotti (2013) apunta que para que se produzca este cambio en la mentalidad de los ciudadanos, estos deben adquirir una conciencia crítica que les haga responsables de sus acciones y que entiendan cuáles son los problemas que afectan a la sociedad.

La investigación que aquí se muestra resulta especialmente innovadora, pues relaciona esta formación de ciudadanos globales con la psicología positiva. La psicología positiva busca la felicidad de tres maneras: emoción positiva, sentido y compromiso (Seligman, 2016). Dicha psicología guarda una estrecha relación con la EDCG, ya que desde ambas perspectivas se aboga por despertar el sentimiento de responsabilidad en las personas. En este sentido, en base a autores como Morillo (2014) o Galiero (2009), cabe añadir que se debe impulsar un nuevo modelo de ciudadanía global para que la población sea consciente de la realidad social y tenga un compromiso activo en la creación de un mundo más equitativo y solidario. Por tanto, esta propuesta trata de fomentar un bienestar social, lo que hace imprescindible que se propicien en el aula aspectos ligados a la psicología positiva, que en este caso concreto se tratan mediante el programa Aulas Felices de Arguís et al. (2012).

El proyecto Aulas Felices está fundamentado en la psicología positiva y busca compatibilizar "el aprendizaje de los contenidos escolares con el bienestar en las aulas. Sus objetivos principales son dos: incrementar la felicidad en alumnado, profesorado y familias y potenciar el desarrollo personal y social del alumnado" (Bisquerra y Hernández, 2017, p. 60). Cabe destacar, como señalan Body, Ramos, Recondo y Pelegrina (2016), que en España existen otros programas que trabajan la psicología positiva en los centros educativos, por ejemplo: Meditación Fluir (Franco et al., 2011) o TREVA (López-González y Oriol, 2016).

Esta investigación se desarrolla en el contexto educativo español y se centra especialmente en los aspectos del programa Aulas Felices tratados en la asignatura llamada: Valores Sociales y Cívicos (en adelante, VSC), tal y como aparece reflejada en Real Decreto 126/ 2014 de 28 de febrero, por el que se establece el currículo básico de la Educación Primaria y la Orden EDU/519/2014, de 17 de junio, por la que se establece el currículo y se regula la implantación, evaluación y desarrollo de la Educación Primaria en la Comunidad Autónoma de Castilla y León. Sin embargo, a pesar de que se pretendía tratar la EDCG en el área de VSC, esta experiencia no 
quedó limitada a esta asignatura, sino que se trabajó de forma transversal en todo el centro educativo al efectuarse en una Comunidad de Aprendizaje.

Entonces, ¿qué es una Comunidad de Aprendizaje? Valls (2005) esclarece el significado afirmando que una Comunidad de Aprendizaje es un proyecto que busca un cambio social y cultural tanto en el centro educativo como en el entorno. Las Comunidad de Aprendizaje están fundamentadas en los principios del aprendizaje dialógico y busca ofrecer una educación participativa en todos los espacios del centro educativo; de este modo, se propicia la participación desde el proyecto educativo de centro y se pretende que las familias, los voluntarios y el alumnado trabajen unidos.

Este proceso de transformación de un centro educativo en una Comunidad de Aprendizaje, como exponen Elboj, Puigdellívol, Soler y Valls (2005), Ileva consigo una serie de etapas (véase Figura 1).

Figura 1. Fases de la transformación de un centro en CA, basado en Elboj et al. (2005)

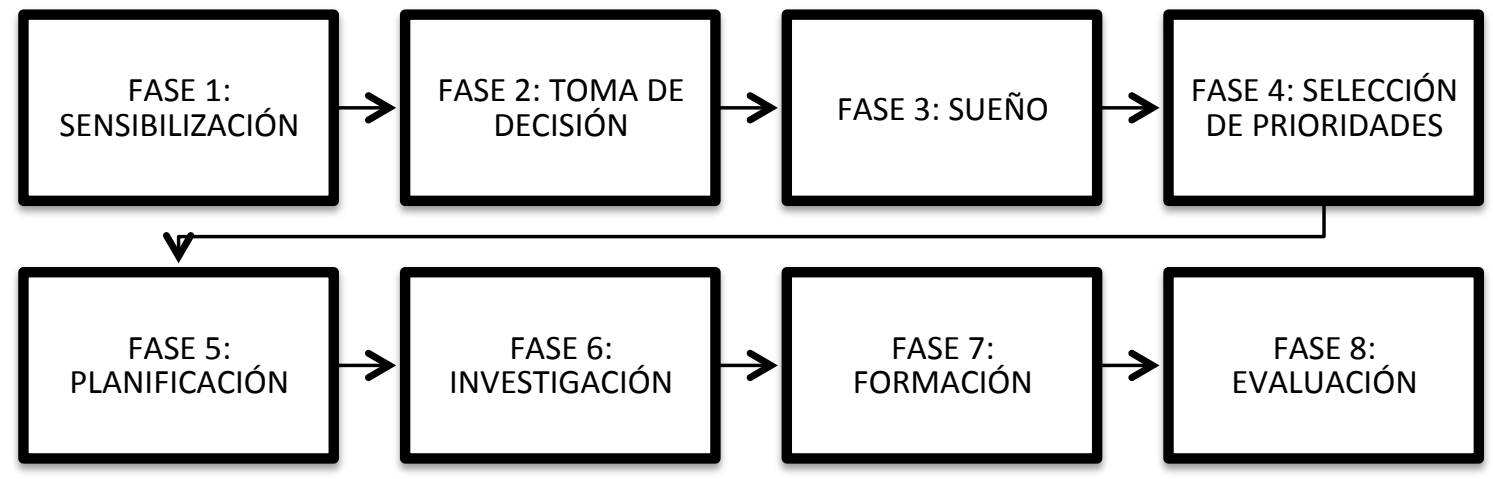

Todas las personas que pertenecen a una Comunidad de Aprendizaje tienen que colaborar con la ejecución de cada una de estas etapas para lograr crear una escuela que busque una mejora educativa. En base a esta concepción de Comunidad de Aprendizaje, se debe añadir que trabajar la EDCG es indispensable en todas las escuelas, pero aún más en una Comunidad de Aprendizaje debido a que todas las personas que forman parte de esta comunidad tratan de crear un colegio para sus hijos que facilite una respuesta educativa basada en la igualdad y que tenga como objetivo mejorar la educación (Elboj et al., 2005). Asimismo, a través de la colaboración de todos los miembros se busca provocar un cambio en la realidad, fundamentándose en principios tan relevantes como la solidaridad, el diálogo y la igualdad. En definitiva, no hay lugar a dudas de la relevancia de la temática presentada en este artículo porque es necesario formar personas capaces de desenvolverse "en un mundo cambiante y complejo como el nuestro, más aún en estos tiempos de crisis e incertidumbre" (Celorio, 2017, p.138). Además, este estudio abarca un tema muy presente en los debates educativos actuales y aporta una visión innovadora en el campo de la investigación tanto de la psicología positiva, como de la EDCG al relacionar el proyecto Aulas Felices con la creación de personas globales y críticas en una Comunidad de Aprendizaje rural.

\section{Objetivos}

- Comprobar si las actividades propuestas basadas en el proyecto Aulas Felices (Arguís et al., 2012) fomentan un cambio de actitudes en el alumnado de $2^{\circ}, 5^{\circ}$ y $6^{\circ}$ de Educación Primaria.

- Despertar en los alumnos el interés por la asignatura de VSC. 
- Crear ciudadanos críticos y globales especialmente desde la asignatura de VSC.

En síntesis, con este estudio se trata de comprobar si la asignatura de VSC cursada en una Comunidad de Aprendizaje rural que emplea el proyecto de psicología positiva Aulas Felices favorece la creación de ciudadanos globales y críticos.

\section{Métodos}

\section{Participantes y contextualización}

La Comunidad de Aprendizaje en la que se realizó este proyecto se encuentra en una zona rural a escasos $15 \mathrm{~km}$ de Segovia capital. Las familias de los estudiantes que acuden al colegio poseen un nivel social y económico medio. El centro educativo consta de dos unidades en Educación Infantil (educandos de tres años, por un lado, y de cuatro y cinco años, por otro) y de cuatro unidades en Educación Primaria (una para $1^{\circ}$, otra para $2^{\circ}$ y las otras dos se dividen en $3^{\circ}$ y $4^{\circ}$, y 5 y $6^{\circ}$ ).

Esta investigación recoge las experiencias realizadas durante el curso 2015/2016 en $2^{\circ}, 5^{\circ}$ y $6^{\circ}$ curso, pues son los niveles en los que la investigadora impartía clase de VSC. En concreto, la muestra con la que se ha trabajado ha sido de 14 discentes, cuatro alumnos de $2^{\circ}$ de Primaria y diez de $5^{\circ}$ y $6^{\circ}$.

\section{Material y metodología didáctica empleada}

El material que empleamos para trabajar en VSC fueron actividades diseñadas específicamente para cada una de las Unidades Didácticas que aparecen marcadas en el programa Aulas Felices. Este proyecto se aplicó a nivel de centro y, especialmente, en la asignatura de VSC. Como se muestra en la Tabla 1, las fortalezas propuestas por Arguís et al. (2012) se encuentran directamente relacionadas con diversos temas transversales y, por tanto, con la asignatura de VSC. Dichas fortalezas tienen su origen en las fortalezas que Seligman (2002) y Peterson y Park (2009) asocian al "buen carácter".

\begin{tabular}{|ll|}
\hline \multicolumn{2}{|c|}{ Tabla 1. Relación entre las Fortalezas y los temas transversales, a partir de Arguís et al. (2012) } \\
\hline FORTALEZAS PERSONALES & TEMAS TRANSVERSALES \\
\hline $\begin{array}{l}\text { Sabiduría y Conocimiento } \\
\text { (Creatividad, Curiosidad, Apertura } \\
\text { mental, Amor por el aprendizaje, } \\
\text { Perspectiva) }\end{array}$ & $\begin{array}{l}\text { Educación para la tolerancia, la paz y la convivencia; Educación } \\
\text { intercultural; Educación para la igualdad entre hombres y mujeres; } \\
\text { Educación ambiental; Educación para la salud; Educación del } \\
\text { consumidor; Educación vial }\end{array}$ \\
\hline $\begin{array}{l}\text { Coraje (Valentía, Perseverancia, } \\
\text { Integridad, Vitalidad) }\end{array}$ & Educación para la tolerancia, la paz y la convivencia \\
\hline $\begin{array}{l}\text { Humanidad (Amor, Amabilidad, } \\
\text { Inteligencia social) }\end{array}$ & $\begin{array}{l}\text { Educación para la tolerancia, la paz y la convivencia; Educación } \\
\text { intercultural; Educación para la igualdad entre hombres y mujeres }\end{array}$ \\
\hline $\begin{array}{l}\text { Justicia (Ciudadanía, Sentido de la } \\
\text { justicia, Liderazgo) }\end{array}$ & $\begin{array}{l}\text { Educación para la tolerancia, la paz y la convivencia; Educación } \\
\text { intercultural; Educación para la igualdad entre hombres y mujeres; }\end{array}$ \\
\hline $\begin{array}{l}\text { Moderación (Capacidad de perdonar, } \\
\text { Modestia, Prudencia, Autocontrol) }\end{array}$ & Educación ambiental; Educación del consumidor; Educación vial \\
\hline $\begin{array}{l}\text { Trascendencia (Apreciación de la } \\
\text { belleza, Gratitud, Esperanza, Sentido } \\
\text { del humor, Espiritualidad) }\end{array}$ & Educación para la tolerancia, la paz y la convivencia; Educación para la \\
\hline
\end{tabular}

Incluso, las fortalezas propuestas por Arguís et al. (2012) están directamente relacionadas con todas las competencias clave vigentes en el currículum escolar, pero 
dirigiremos la mirada en la Tabla 2 hacia las que, por su propia definición y naturaleza, guardan una mayor conexión con la educación en valores.

Tabla 2. Relación entre fortalezas y Competencias Básicas. Fuente: Arguís et al. (2012, p.52).

\begin{tabular}{|c|c|c|c|}
\hline Competencias & $\begin{array}{l}\text { AUTONOMÍA } \\
\text { E INICIATIVA } \\
\text { PERSONAL }\end{array}$ & SOCIAL Y CIUDADANA & $\begin{array}{c}\text { APRENDER A } \\
\text { APRENDER }\end{array}$ \\
\hline $\begin{array}{c}\text { Fortalezas Per- } \\
\text { sonales }\end{array}$ & $\begin{array}{l}\text { Creatividad } \\
\text { Curiosidad } \\
\text { Apertura mental } \\
\text { Amor por el aprendizaje } \\
\text { Valentía } \\
\text { Perseverancia } \\
\text { Vitalidad } \\
\text { Modestia } \\
\text { Prudencia } \\
\text { Autocontrol } \\
\text { Apreciación de la belleza } \\
\text { Esperanza } \\
\text { Sentido del humor } \\
\text { Espiritualidad }\end{array}$ & $\begin{array}{l}\text { Perspectiva } \\
\text { Integridad } \\
\text { Amor } \\
\text { Amabilidad } \\
\text { Inteligencia social } \\
\text { Ciudadanía } \\
\text { Sentido de la justicia } \\
\text { Liderazgo } \\
\text { Capacidad de perdonar } \\
\text { Gratitud }\end{array}$ & $\begin{array}{l}\text { Creatividad } \\
\text { Curiosidad } \\
\text { Apertura mental } \\
\text { Amor por el aprendizaje } \\
\text { Perspectiva } \\
\text { Perseverancia } \\
\text { Ciudadanía } \\
\text { Autocontrol }\end{array}$ \\
\hline
\end{tabular}

Al realizar el estudio en una Comunidad de Aprendizaje todas las actividades elaboradas estaban basadas en el aprendizaje dialógico. Tomando como referencia a Aubert et al. (2010) y Elboj et al. (2005), durante el desarrollo de estas Unidades Didácticas se ha fomentado el diálogo basado en la igualdad y la inteligencia cultural. A su vez, se pretendió trabajar la dimensión instrumental y el desarrollo de valores tan importantes como la solidaridad, el respeto y la responsabilidad.

Las actividades se realizaban a través del aprendizaje cooperativo, pues el alumnado debía trabajar en equipo para lograr un resultado óptimo. Para emplear este aprendizaje participativo se planteaban constantes ejercicios de debate sobre los vídeos que se iban viendo en los ejercicios propuestos; esto es así porque se buscaba fomentar momentos en el aula en los cuales los estudiantes pudieran compartir sus puntos de vista. Además, se pretendía "crear un ambiente donde los alumnos pueden practicar habilidades sociales y de liderazgo [...] y, por último, proporcionar una satisfactoria experiencia de aprendizaje" (García-Valcárcel, Basilotta y López, 2014, p.66).

Como se ha destacado anteriormente, una amplia variedad de los ejercicios propuestos consistía en la visualización de cortometrajes para posteriormente debatir en grupo sobre ellos. Con estas actividades, se emplearon las Tecnologías de la Información y la Comunicación (TIC) porque, como afirma Gutiérrez (2003), los alumnos actualmente son nativos digitales y emplear estas tecnologías ayuda a captar su atención y a motivarles.

Cabe añadir que se empleó una evaluación formativa porque se consideró que era la más adecuada para "la mejora de los procesos de aprendizaje del alumno, el perfeccionamiento del docente y los procesos de enseñanza-aprendizaje que tienen lugar en un contexto educativo" (López-Pastor, 2009, p.94). Este modelo de evaluación se implementó mediante la retroalimentación que se proporcionaba a cada uno de los escolares en cada debate, al poner en común con otros profesores las actividades realizadas con el alumnado y al emplear el cuaderno de campo para poder mejorar nuestra actuación como docentes. 


\section{Metodología para la investigación}

La presente investigación ha utilizado una metodología cualitativa porque consideramos que responde adecuadamente a los objetivos propuestos anteriormente (Rodríguez, Gil y García, 1999). Esto es así porque la investigación cualitativa "produce datos descriptivos: las propias palabras de las personas, habladas o escritas, y la conducta observable" (Taylor y Bogdan, 2010, p.20). Y este es el material que empleamos en el estudio: la conducta observable de los participantes y las anotaciones que realiza la investigadora en su cuaderno de campo.

\section{Acceso al campo}

El campo abarca "el contexto físico y social en el que tienen lugar los fenómenos objeto de la investigación" (Rodríguez et al., 1999, p.103). En este estudio concreto el acceso al campo ha resultado sencillo, ya que la persona encargada de esta investigación se encontraba realizando sus prácticas académicas del Grado en Educación Primaria en esta Comunidad de Aprendizaje.

\section{Técnicas e instrumentos de recogida de datos}

La técnica escogida para la obtención de datos es la observación participante, la cual se aplica para "obtener datos sobre cualquier realidad social; si bien en la práctica la observación participante se emplea para obtener datos sobre realidades a las que resulta difícil aplicar otro tipo de técnicas" (Guasch, 1996, p. 35). En este caso concreto, se pensó que el empleo de esta técnica era el más adecuado porque al impartir clase en estos cursos era muy sencillo para la investigadora recopilar datos y después reflejarlos en su cuaderno de campo. La investigadora recogió la información en un cuaderno o diario de clase pues, acorde con Zabalza (2004), con esta técnica se consigue que el profesorado anote información sobre lo que va aconteciendo en las clases. Durante la realización de la observación participante se tuvieron presentes diversos principios éticos asegurando a todos los participantes el anonimato y la confidencialidad. Incluso, para garantizar la transparencia se proporcionó al tutor de prácticas del centro educativo, al tutor de prácticas de la universidad y a la persona encargada de la dirección del centro, las anotaciones del cuaderno de campo con la finalidad de otorgar rigurosidad a esta investigación teniéndose como referencia los criterios propuestos por Guba (1989).

\section{Procedimiento de análisis de la información}

La información recopilada en el cuaderno de campo se analiza cualitativamente a través de la elaboración de diversas categorías (Kvale, 2011). Autores como Taylor y Bogdan (2010), Álvarez-Gayou (2006), o Rodríguez et al. (1999) afirman que el análisis cualitativo requiere de tres etapas principales: (1) fase de identificar los temas, los conceptos clave o las proposiciones; (2) fase que tiene lugar después de recoger la información y comprende la codificación de los datos, y (3) fase final en la cual el investigador intenta comprender los datos teniendo en cuenta el contexto en el que fue recogida. Tomando como base las tres etapas anteriormente descritas, se han establecido una serie de categorías para analizar la información recogida en el cuaderno de campo. A continuación, se presentan las categorías que se han realizado en función de los valores o virtudes trabajados:

- Apertura mental

- Amor por la naturaleza

- Amor y comprensión 
- Amabilidad

\section{Resultados}

A continuación, se muestran los resultados obtenidos en base a las categorías establecidas anteriormente.

\section{Apertura mental}

Al trabajar la fortaleza de la apertura mental, se consiguió hacer a los estudiantes reflexionar y "pensar sobre las cosas y examinar todos sus significados y matices. No precipitarse al sacar conclusiones, sino tras evaluar cada posibilidad" (Arguís et al., 2012).

Los educandos participantes trabajaron la apertura mental utilizando la actividad del "Mikado", la cual consistía en que cada alumno contara un problema, y los demás le sugirieran soluciones. Con los alumnos de $5^{\circ}$ y $6^{\circ}$ se realizó una actividad llamada "rincones de opinión", la cual consiste en que los estudiantes exponen sus problemas y posteriormente, se seleccionan los problemas que más interesen para intentar resolverlos. Los estudiantes se sitúan por rincones, tratando un problema diferente en cada rincón y pudiéndose cambiar de rincones para aportar sugerencias en los demás grupos. Posteriormente, cuando tienen la respuesta se exponen en grupo y se valora entre toda la clase fomentando la participación del alumnado y la disparidad de puntos de vista.

Los problemas planteados por los estudiantes fueron, por ejemplo: el desinterés en hacer los deberes, los problemas que tienen en clase con un estudiante que interrumpe continuamente y cómo ayudar a un estudiante de otro país que no hablaba español en el aula porque le veían algo triste. Durante el debate grupal se decido tratar la cuestión de la incorporación de un nuevo estudiante al centro educativo; en este caso, los estudiantes proponían ayudarla durante la realización de los ejercicios, jugar con ella en el recreo e intentar que se sintiera cómodo a pesar de sus diferencias con el idioma.

Dichas propuestas de actuación se llevaron a la práctica, lo que permitió comprobar a lo largo del curso cómo el estudiante empezaba a dominar la lengua, las asignaturas y cómo gracias a las atenciones de sus compañeros empezó a sentirse más feliz en el centro educativo. Por ello, el punto de vista del docente recogido en el diario de clase afirma que tratar esta fortaleza hace "reflexionar a los niños permitiéndoles tanto adquirir conocimientos positivos para su formación integral y equilibrada, como valorar las opiniones de los demás, darse cuenta de que hay otros puntos de vista y la importancia de ayudar a los demás".

En síntesis, la apertura mental desarrollada a través de actividades que incitan a que los discentes sean capaces de valorar diferentes situaciones problemáticas que afectan a la sociedad en la que viven e intentar proponer soluciones de forma cooperativa favorece la creación de una ciudadanía global.

\section{Amor a la naturaleza}

Otro aspecto que se trató desde el área de VSC es el amor por la naturaleza; para ello, se realizaron actividades en torno a dos temáticas: (1) la importancia de cuidar a los animales y (2) los efectos de la contaminación producida por los desechos humanos. 
La necesidad de cuidar a los animales fue tratada mediante el visionado de unos cortometrajes que pretendían sensibilizar al alumnado y provocar en ellos reflexiones que fueron comentadas en un debate posterior para finalmente elaborar una serie de pautas para cuidar bien a un animal. Como se recoge en el cuaderno de campo "los niños reflexionaron sobre el tema y participaron activamente"; asimismo, algunos estudiantes añadían que la docente era "un poco dramática por las cosas que les estaba contando, pero que entendían que era muy importante cuidar a los animales".

Los efectos provocados por la contaminación causada con los malos hábitos humanos se mostraron a los estudiantes mediante un vídeo en el que podían observar la contaminación existente en el mar, la cual provoca la muerte de plantas marinas y animales. Además, se enseñaron una serie de imágenes en las que podían ver cómo los animales se veían afectados por esta contaminación que afecta al mundo en el que viven. Durante estas actividades, el docente fue estableciendo un debate con los discentes haciendo hincapié en la idea de no contaminar ni la tierra, ni el agua y la importancia de llevar unos hábitos de vida saludables para el medio ambiente.

Una evidencia de que al trabajar estas actividades en los alumnos se despertaran actitudes que mostraran interés por realizar acciones contra la contaminación y para ayudar a los animales, se pudo apreciar en unos dibujos que elaboraron los discentes. En estas ilustraciones se apreciaban personas ayudando a los animales, cuidando los océanos y reciclando. Resulta interesante resaltar la ilustración elaborada por una alumna de $2^{\circ}$ de Primaria en la cual plasmó el centro educativo con contenedores de colores para reciclar y carteles con mensajes que incitaban al desarrollo sostenible y a la protección de los animales.

Como reflexión final, en el cuaderno de campo se recoge que los ejercicios propuestos "funcionaron perfectamente, pues los niños rápidamente entendieron la importancia de no tirar desechos al mar y la necesidad de actuar y reciclar la basura en los contenedores indicados de reciclaje". Por tanto, el resultado de los ejercicios propuestos fue exitoso llegando a llamar la atención de la investigadora en "la facilidad con la que entendieron las cosas y sobre todo su reacción cuando vieron las imágenes de los animales dañados por culpa de estos desechos".

\section{Amor y comprensión}

El proyecto Aulas Felices destaca el valor del amor y la comprensión, el cual se trabajó con el alumnado participante de $2^{\circ}, 5^{\circ}$ y $6^{\circ}$ a través de unas actividades diseñadas a tal fin. En concreto, se visualizaron una serie de cortometrajes con los que se pretende valorar "las relaciones cercanas con otras personas, en particular aquellas en las que el afecto y el cuidado son mutuos. Sentirse cercano a otras personas" (Arguís et al., 2002, p.185) y posteriormente, se fue realizando un pequeño debate sobre cada uno de los vídeos propuestos.

Durante las reflexiones en común en el aula los estudiantes mostraban que habían comprendido la importancia de tratar bien a las personas, especialmente a nuestros seres queridos. Uno de los aspectos que manifiestan actitudes ligadas a la ciudadanía global se pudo apreciar en la última conclusión propuesta por los estudiantes, la cual fue que como personas debían ser comprensivas y ayudarse de manera cooperativa para superar todas las adversidades y enfrentarse a las injusticias. En base a este discurso generado, se puede apreciar que la aplicación del debate en el aula tiene resultados muy positivos; esto es así porque, como se apunta en el cuaderno de campo, "las aportaciones que realizan los niños son muy enriquecedoras y les hacen reflexionar, especialmente a los alumnos de $5^{\circ}$ y $6^{\circ}$ ". 
Gracias a la realización de los ejercicios propuestos, los cuales "tuvieron mucho éxito entre los alumnos y funcionaron a la perfección", se consigue despertar en los estudiantes el sentido crítico y reflexivo. En este sentido, y partiendo del enfoque de la EDCG, se pretende favorecer a la creación de ciudadanos globales que sean conscientes de los problemas existentes en la sociedad e intenten transformar el mundo en un lugar más justo y solidario.

\section{Amabilidad}

Al trabajar el valor de la amabilidad, se pretendía trasmitir a los escolares la idea de actuar bien con los demás, "ayudar y cuidar de otras personas" (Arguís et al., 2012, p.198). Los ejercicios planteados seguían la misma línea que al trabajar otros valores, es decir, empleamos las TIC para ver cortometrajes, los cuales posteriormente nos permiten establecer un debate y reflexionar sobre la temática que tratamos en el aula.

En esta misma sesión los educandos se fijaron en "unas fotos que había en la biblioteca de los niños de la escuela de Larabanga (Ghana)"; gracias a estas imágenes y a la inquietud que despertaron en los discentes, el docente refleja en el cuaderno de campo lo siguiente: "consideré oportuno trabajar con ellos la necesidad de ayudar a los demás y les hice comprender que se puede ser feliz con muy poco".

Durante la asamblea colectiva, los educandos trataron temas tan importantes como la importancia de ser amables, ayudar, ser personas cívicas y respetar a los demás. La idea final con la que se concluyó el debate fue que, si nosotros colaboramos tratando de ser más amables y comprometidos probablemente contagiemos a otras personas intentando, de este modo, crear una ciudadanía que convierta el mundo en un lugar más amable y humano. Por consiguiente, estas ideas que se trataron en el diálogo permiten percibir, como muestra la investigadora en el cuaderno de campo, que "las actividades propuestas funcionaron correctamente".

\section{Discusión}

En base a los resultados obtenidos, se puede destacar que tratar la apertura mental del alumnado favorece a la consecución de uno de los fines propuestos en la EDCG: ayudar a los estudiantes a descubrir y sensibilizarse ante la realidad, a valorar diferentes situaciones y a "movilizarse ante los problemas fundamentales de la humanidad, en especial los que causan el sufrimiento de los excluidos de este mundo nuestro" (De la Calle, Rodríguez, Ruíz y Torrego, 2003, p.1).

Cabe añadir que la EDCG "promueve valores y actitudes relacionados con la solidaridad y la justicia social. Implica ser un sujeto activo comprometido con los problemas locales y globales, relacionados con la paz, los derechos humanos y la justicia social" (Mesa, 2014, p.104). En relación con esta idea, se trató de sensibilizar a los estudiantes participantes mediante el trabajo del amor por la naturaleza al situarles ante una problemática que afecta directamente a la sociedad en la que viven tratando así, de formar ciudadanos globales y críticos que defiendan un Desarrollo Sostenible. En este sentido, se pretende que las personas participantes comprendan la importancia de modificar su propio estilo de vida provocado por la actual sociedad de consumo intentando potenciar un cambio en sus hábitos que aboguen por un Desarrollo Sostenible y Humano (Celorio, Celorio y López, 2012).

Al tratar de despertar el amor y la comprensión se pretendía que los estudiantes fueran conscientes actuar adecuadamente tratando de ayudar a los demás. En este aspecto, González (2000) comenta que en la escuela deberían extenderse estas 
sesiones en las que se incita al alumnado a desarrollar un pensamiento crítico y reflexionar, pues se deben alfabetizar a los estudiantes para que desarrollen

...una adecuada formación en valores críticos y reflexivos y potencie estrategias de acceso a la información individual y grupal, desarrollando un sentido autónomo de la educación, ayudándoles a comprender el mundo y su mundo, su cultura y su relación con esa cultura, lo que les gusta y lo que no les gusta, llevándolos a pensar lo que puede y debe ser la igualdad y la equidad. Es decir, aportar herramientas para pensar críticamente la realidad y su realidad inmediata y no prohibir sin formación alguna, ciertos mensajes. (González y Muñoz, 2002, p. 220)

Durante el trabajo de la amabilidad con los escolares participantes se puede apreciar que, en consonancia con las ideas que emergen de la EDCG, resulta imprescindible enseñar a los estudiantes la importancia de cooperar con los demás. Incluso, la prioridad de abogar por el Buen Vivir, es decir, por transformar la sociedad intentando adaptarse "a un mundo con menos recursos y que esa adaptación no sea caótica sino ordenada y justa” (Rodríguez-Marín, Fernández-Arroyo y García, 2015, p. 39).

Por otro lado, se debe señalar que con este estudio se han obtenido los resultados esperados y que el primero de los objetivos propuestos se ha cumplido. Esto es así porque las sesiones han funcionado adecuadamente haciendo reflexionar al alumnado participante e intentando forjar en ellos una conciencia crítica que abogue por el bienestar social.

En cuanto al segundo de los objetivos fijados anteriormente, se ha logrado aumentar el interés de los estudiantes por la asignatura de VSC. Los buenos resultados obtenidos en esta experiencia permiten afirmar que la metodología empleada, en la cual se utilizaban las TIC y se pretendía despertar este interés "a través de curiosidades, anécdotas, problemas de la actualidad, cuestiones que tengan relación con la vida cotidiana, etc." (Mellado, 1996, p. 295), aviva la motivación de los discentes por la clase de VSC.

El último objetivo se fue cumpliendo a través de las tareas empleadas en la asignatura de VSC porque tanto con los valores trabajados, como a través de los momentos de reflexión y diálogo en el aula, se lograron fomentar actitudes propias de una ciudadanía global crítica. En esta línea, se llega a la conclusión de que es imprescindible que se emplee la EDCG en todas las áreas, pues como afirma (Lemke, 2006) hay que enseñar y esforzarse en "formar buenos ciudadanos globales con valores morales más humanos" (p.7).

Se debe resaltar que la principal limitación del estudio se encuentra en el número de estudiantes que asistían a las clases de VSC, pues el centro rural no cuenta con muchos alumnos lo cual se ve más reducido aún al tratarse de una asignatura optativa. El hecho de no contar con más discentes y de no haber continuado el estudio indagando en más fortalezas o valores debido a la finalización del periodo de prácticas de la investigadora hace que este estudio se vea limitado y no permita realizar generalizaciones; sin embargo, esto no impide vislumbrar los resultados positivos en las actitudes de los estudiantes al tratar de educar para la ciudadanía global mediante el proyecto Aulas Felices.

La principal fortaleza que encontramos en este estudio es la posibilidad de fomentar el bienestar en las aulas y, por extensión, en la sociedad mediante la aplicación de proyectos de psicología positiva y la EDCG. Una futura línea de investigación en relación con la EDCG sería investigar si se trabaja de forma transversal y qué 
resultados se pueden apreciar en los comportamientos de los estudiantes a medida que van adquiriendo conocimientos relacionados con esta temática.

En conclusión, en este estudio realizado se han obtenido los resultados deseados y se ha vislumbrado la importancia de que en todas las asignaturas se contribuya a formar ciudadanos críticos, pues es necesario que se creen personas libres que sean capaces de comprender el mundo para transformarlo en un lugar más equitativo, justo y solidario.

\section{Referencias}

Álvarez-Gayou, JL. (2006). Cómo hacer investigación cualitativa. Fundamentos y metodología. Barcelona: Paidós.

Andreotti, V. (2013). Conhecimento, Escolarização, Currículo E a Vontade De 'Endireitar' a Sociedade Através Da Educação. Revista Teias, 14(33), 215-27.

Argibay, M. y Celorio, G. (2005). La Educación para el Desarrollo. Vitoria: Gobierno Vasco.

Arguís, R., Bolsas, AP., Hernández, S. y Salvador, M. (2012). Programa Aulas Felices. [WebCite ${ }^{\circledR}$ at http://www.webcitation.org/6xaZiBCp9]

Aubert, A., Flecha, A., Garcia, C., Flecha, R. y Racionero, S. (2010). Aprendizaje dialógico en la sociedad de la información. Barcelona: Hipatia.

Body, L., Ramos, N., Recondo, O. y Pelegrina, M. (2016). Desarrollo de la Inteligencia Emocional a través del programa mindfulness para regular emociones (PINEP) en el profesorado. Revista interuniversitaria de formación del profesorado, 87, 47-59.

Boni, A. (2014). Un análisis de los discursos institucionales en la cooperación y la educación desde la perspectiva de la Educación para la Ciudadanía Global. Reflexiones a partir del caso español. Sinergias, 1, 101-115.

Boni, A. y León, R. (2013). Educación para una ciudadanía global: una estrategia imprescindible para la justicia social. Informe Intermón Oxfam: La Realidad de La Ayuda 2012, 215-239.

Bisquerra, R. y Hernández, S. (2017). Psicología positiva, educación emocional y el programa de aulas felices. Papeles del psicólogo 38(1), 58-65.

Calvo, A. (2017). The state of development education in Spain: Initiatives, trends and challenges. International Journal of Development Education and Global Learning, 9(1), 18-32.

Celorio, JJ. y Celorio, G. (2011). ¿Educación? para el ¿desarrollo? Pueblos. Revista de información y Debate, 46, 1-3.

Celorio, G. (2017). Educación crítica y transformadora. Marco teórico-pedagógico para integrar la soberanía alimentaria con enfoque de género en los centros de secundaria. Barcelona: VSF Justicia alimentaria global.
Celorio, G., Celorio, JJ. y López, A. (2012). La Educación para el Desarrollo en la Universidad. Reflexiones en torno a una práctica transformadora. Bilbao: Hegoa.

De la Calle, MJ., Rodríguez, M., Ruíz, E. y Torrego, L. (2003). La Educación para el Desarrollo en el marco educativo. Valladolid: GEDEPAZ. DOE. Universidad de Valladolid.

Davies, I., Evans, M., \& Reid, A. (2005). Globalising Citizenship Education? A Critique of "Global Education" and "Citizenship Education". British Journal of Educational Studies, 53(1), 6689.

Díaz, A. (2005). La educación en valores: Avatares del currículum formal, oculto y los temas transversales. Revista Electrónica de Investigación Educativa, 8(1), 1-15.

Elboj, C., Puigdellívol, I., Soler, M. y Valls, R. (2005). Comunidades de aprendizaje. Transformar la educación. Barcelona: Graó.

Franco, C., Mañas, I., Cangas, AJ., Moreno, E. y Gallego, J. (2011). Reducing teachers psychological distress through mindfulness training. Spanish Journal of Psychology, 13(2), 655-666.

Galiero, M. (2009). Educar para una ciudadanía global: construir un mundo justo desde la escuela. Barcelona Intermón Oxfam.

García-Valcárcel, A, Basilotta, V. y López, C. (2014). Las TIC en el aprendizaje colaborativo en el aula de Primaria y Secundaria. Comunicar, 42, 65-74.

González, J. (2000). Variables de la educación en comunicación. En J. Pérez, Comunicación y educación en la sociedad de la información. Madrid: Paidós.

González, M. y Muñoz, J. (2002). La formación de ciudadanos críticos. Una apuesta por los medios. Teoría de la Educación. Revista Interuniversitaria, 14, 207-233.

Guasch, O. (1996). Observación participante. Cuadernos metodológicos. Madrid: CIS.

Guba, EG. (1989). Criterios de credibilidad en la investigación naturalista. En J. Gimeno y A. Pérez Gómez (Eds.). La enseñanza: su teoría y su práctica (148-165). Madrid: Akal.

Gutiérrez, A. (2003). Alfabetización digital. Algo más que ratones y teclas. Barcelona: Gedisa. 
Kvale, S. (2011). Las entrevistas en Investigación Cualitativa. Madrid: Morata.

Lemke, JL. (2006). Investigar para el futuro de la educación científica: nuevas formas de aprender, nuevas formas de vivir. Enseñanza de las ciencias: revista de investigación y experiencias didácticas, 24(1), 5-12.

López-González, L., \& Oriol, X. (2016). The relationship between emotional competence, classroom climate and school achievement in high school students. Culture Education, 28,130-156.

López-Pastor, VM. (Coord.). (2009). Evaluación Formativa y Compartida en Educación Superior. Propuestas, técnicas, instrumentos y experiencias. Madrid: Narcea.

Mellado, V. (1996). Concepciones y prácticas de aula de profesores de ciencias, en formación inicial de primaria y secundaria. Enseñanza de las ciencias: revista de investigación y experiencias didácticas, 14(3), 289-302.

Mesa, M. (2014). La educación para la ciudadanía global en el contexto actual. En J. L. Pastoriza (ed.), Educar para la ciudadanía global. Experiencias, herramientas y discursos para el cambio social. Vigo: Fundación Isla Couto.

Monk, D. (2014). Introducing Corporate Power to the Global Education Discourse. Policy \& Practice: A Development Education Review, 19, 33-51.

Morillo, BF. (2014). Reconstruir la ciudadanía en un entorno de incertidumbre: una tarea educativa. Postconvencionales: ética, universidad, democracia, 7, 19-31.

Murray, S. (2006). Editorial. Policy \& Practice: A Development Education Review, 3, 1-4.

Ortega, ML. (2007). Estrategia de Educación para el Desarrollo de la cooperación española. Madrid: Ministerio de Asuntos Exteriores y de Cooperación. Secretaría de Estado de Cooperación Internacional Dirección General de Planificación y Evaluación de Políticas para el Desarrollo.

Ortega-Quevedo, V., Santamaría-Cárdaba, N. y Gil-Puente, C. (2017). La evaluación formativa en una secuencia de enseñanza aprendizaje de Ciencias Experimentales. Revista Infancia, Educación y Aprendizaje, 3(2), 203-209.

Pérez, A., Casanova, P., Vega, D., Álvarez, L., Durán, F. y Pablos, L. (2009). Capacidades del alumnado de primaria para una Educación Física integral en la LOE. Características psicopedagógicas. Revista digital EF Deportes, 135. [WebCite ${ }^{2}$ at http://www.webcitation.org/6xaaByEp9]

Reysen, S., \& Katzarska-Miller, I. (2013). A Model of Global Citizenship: Antecedents and Outcomes. International Journal of Psychology, 48(5), 858-70.

Rodríguez, G., Gil, J. y García, E. (1999). Metodología de la investigación cualitativa. Málaga: Aljibe.

Rodríguez-Marín, F., Fernández-Arroyo, J. y García, JE. (2015). El huerto escolar ecológico como herramienta para la educación en y para el decrecimiento. Investigación en la escuela, 86, $35-48$
Sainz de Murieta, J. (2016). El papel de las Enseñanzas Técnicas Universitarias en la Cooperación Universitaria al Desarrollo y en la formación de ciudadanía global y transformadora. IKASTORRATZA. e-Revista de Didáctica,17, 1-7.

Seligman, ME. (2016). Florecer: La nueva psicología positiva y la búsqueda del bienestar. México: Oceano.

Smith, W., Fraser, P., Chykina, V., Ikoma, S., Levitan, J., Liu, J., \& Mahfouz, J. (2016). Global Citizenship and the Importance of Education in a Globally Integrated World. Globalisation, Societies and Education, 15(5), 648-65.

Starkey, H. (2012). Human Rights, Cosmopolitanism and Utopias: Implications for Citizenship Education. Cambridge Journal of Education, 42(1), 21-35.

Taylor, SJ. y Bogdan, R. (2010). Introducción a los métodos cualitativos de investigación. Barcelona: Paidós.

Valls, R. (2005). Los educadores y las educadoras sociales en las Comunidades de Aprendizaje. Revista de Educación Social, 4. [WebCite® at http://www.webcitation.org/6xaaJkBN0]

Zabalza, MA. (2004). Diarios de clase. Un instrumento de investigación y desarrollo profesional. Madrid: Narcea. 